\title{
ABSORPTION COLUMNS WITH BRIGHT X-RAY SOURCES NEAR THE GALACTIC CENTER: \\ - MASS ESTIMATION IN THE GALACTIC CENTER REGION
}

\author{
MASAAKI SAKANO, MAMIKO NISHIUCHI, YOSHITOMO MAEDA, \\ KATSUJI KOYAMA AND JUN YOKOGAWA \\ Devartment of Physics, Kyoto University, Sakyo, Kyoto, Japan
}

\begin{abstract}
.
We report the column density distribution to the Galactic Center region using the $\mathrm{X}$-ray binary observations with the $\mathrm{X}$-ray satellite $A S C A$, and demonstrate a new method of the total mass determination near the Galactic Center. The column densities are given by a simple form as a function of the angular distance from the Galactic Plane. Assuming a disklike mass distribution of $500 \mathrm{pc}$ radius, we estimate the total mass to be $\sim 6 \times 10^{7} M_{\odot}$.
\end{abstract}

\section{Introduction}

Many efforts for measuring the intersteller gas distribution in the Galactic Center region have been done with the radio, infrared and gamma-ray observations. The estimated values, however, show a large scatter from $10^{7}$ to $10^{8}$ of solar mass. Therefore new and independent methods would be very important.

We propose here a new method to determine the mass distribution using $\mathrm{X}$-ray absorptions. So far, many X-ray sources, most are transient X-ray binaries, have been observed from the direction of the Galactic Center. If these X-ray sources are distributed isotropically around the Galactic Center, the column densities to these sources would give much information on the distribution of the galactic center gas. This means potentiality to determine the total mass and mass distribution using the spectra of the $\mathrm{X}$-ray sources. This paper demonstrates this capability with the $A S C A$ satellite. Here we assume the distance to the Galactic Center to be $8.5 \mathrm{kpc}$. 


\section{Results}

$A S C A$ has detected 8 bright $\mathrm{X}$ ray stars in the region, $\left|l_{I I}\right|<1^{\circ}$ : A1742-289 (Maeda et al., 1996), 1E1743.1-2843, 1E1740.7-2942 (Sakano et al., 1997), GRS 1741.9-2853, A1742-294, GRO J1744-28 (Dotani et al., 1996), SLX 1737-282, SLX 1735-269 (David et $a l ., 1997)$. We have systematically analyzed their X-ray spectra and determined rather accurate column densities for the first time.

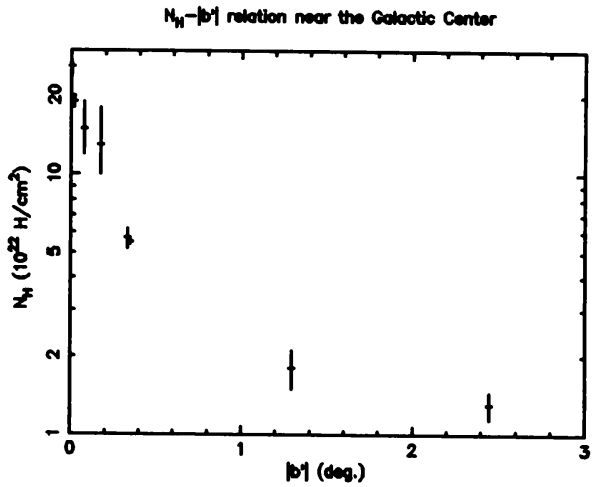

Figure 1. The column densities of X-ray stars as a function of the angular distance from the Galactic Plane.

Together with the previous results, we plot the column densities on Fig. 1 against the angular distance from the Galactic Plane $\left(\left|b^{\prime}\right|=\mid b+\right.$ $\left.0.0460^{\circ} \mid\right)$. We see rather smooth profile in Fig. 1, which suggests that the absorbing gas is isotropically distributed, and most of the selected X-ray sources are closely distributed around the Galactic Center.

We therefore fit the profile on Fig. 1 to a Galactic Center disk model of $\sim 500 p c$ radius with the density depending only on the distance from the Galactic Plane $\left(\left|b^{\prime}\right|=\left|b+0.0460^{\circ}\right|\right)$. The foreground absorption is assumed due to a disk of $100 \mathrm{pc}$ thickness with constant density of $1 \mathrm{H} / \mathrm{cm}^{3}$. As a result, we estimated the total mass of this region to be $6 \times 10^{7} M_{\odot}$.

\section{Discussion}

Since X-ray absorption is mainly due to the oxygen atoms, our new method of estimation of mass distribution is quite independent of others. Our estimated total mass near the Galactic Center is consistent with the value of $<6 \times 10^{7} M_{\odot}$ derived from $\gamma$-rays (Blitz et al., 1985) and infrared (Cox \& Laureijs, 1989), but is inconsistent with that derived from the CO observations (>10 $0^{8} M_{\odot} ;$ Bania 1986$)$. Recently Oka et al. (1997) proposed the radio results to be $(2-6) \times 10^{7} M_{\odot}$, assuming pressure bounded clouds instead of self-gravitationally bounded clouds. This value is consistent with our result. Thus we are confident that the new method is potentially reliable.

\section{References}

Bania, T. (1986), ApJ, 308, 868

Blitz, L. et al. (1985), $A \& A, 143,267$

Cox, P. \& Laureijs, R. (1989), IAU symp.

136: The Center of the Galaxy, p.121

David, P. et al. (1997), $A \mathcal{G} A, 322,229$
Dotani, T. et al. (1996), IAU Circ., No.6337

Maeda, Y. et al. (1996), PASJ, 48, 417

Oka, T. et al. (1997), ApJ, in press

Sakano, M. et al. (1997), in preparation 